\title{
Essential fatty acids in the perinatal period: short- and long-term effects in
} a rat model

\author{
Marina Korotkova
}

\section{Introduction}

Early infant nutrition is one of the most powerful environmental factors that determine early growth and development. Nutritional factors during a sensitive perinatal period may lead to developmental adaptations proposed to programme for pathological conditions later in life. Among the nutritional factors, essential fatty acids (EFA) and their derivatives play a fundamental role in optimal foetal and postnatal development as structural elements of membranes and as functional modulators. Nevertheless, whether the EFA in general or the n-6 or n-3 EFA specifically are required for optimal early metabolism and long-term consequences are poorly understood. In animals and humans, variations in the levels of EFA and in the ratio of n-6 to n-3 EFA affect growth, neural development and immune responsiveness of the offspring, and may also have further effects later in life. Leptin is an adipose tissue-derived hormone that regulates food intake and energy expenditure, and is involved in several physiological and pathological processes, including in the perinatal period. During early development, leptin is produced by the placenta and by foetal and neonatal adipose tissues, and is also provided via maternal milk. In humans, circulating leptin levels in early life predict weight gain later in infancy and leptin level is important for normal development and health. Variation in the type of dietary fat during pregnancy and lactation may modulate foetal and neonatal growth and development by leptin-associated mechanisms.

\section{Experimental design}

An experimental rat model was used, with the main approach of feeding a food that was either depleted or enriched in specific EFA during late gestation and throughout lactation. The rats were fed either a control or an EFA-deficient diet $(1,2)$, a diet containing both n-6 and n-3 EFA, an n-3 EFA-enriched diet or an n-6 EFA-enriched diet $(3,4)$. The fatty acid (FA) composition of serum phospholipids, milk total lipids, and total lipids and phospholipids of inguinal white adipose tissue (WAT) were analysed. Body weight, length, weight of inguinal WAT and adipocyte cell size were evaluated in offspring at 1 and 3 weeks of age. Leptin concentrations in serum and milk were measured and leptin mRNA expression was analysed in WAT. To investigate the long-term consequences of dietary EFA during the perinatal period, the offspring were weaned onto ordinary chow and followed up until adult age. Body weight was recorded, and food intake, blood pressure, plasma insulin and glucose levels were evaluated at 28 weeks of age.

\section{Results and discussion}

\section{Changes in fatty acid composition}

Variations in dietary intake of EFA during pregnancy and lactation induced rapid and marked changes in FA composition in maternal milk and in serum and WAT phospholipids in rat offspring, as well as changes in serum leptin levels and leptin mRNA expression. A deficiency of both n-6 and n-3 EFA in the maternal diet resulted in decreased serum leptin levels in the offspring (1) associated with reduction in both the amount of WAT and the leptin mRNA expression in WAT (2). In addition, milk leptin levels were higher in the EFA-deficient dams than in the controls at 3 weeks of lactation (2). The deficiency of EFA during the perinatal period was associated with reduced body weight of rat offspring at 3 weeks of age (2), followed by an accelerated weight gain in the adult rats. Starting from 12 weeks of age the rat offspring receiving an EFA-deficient diet perinatally had a significantly increased body weight compared with the controls and this difference was more pronounced at 30 weeks of age $(p<0.05$, Fig. 1). Restriction of dietary EFA during pregnancy and lactation induced developmental adaptations 
A.

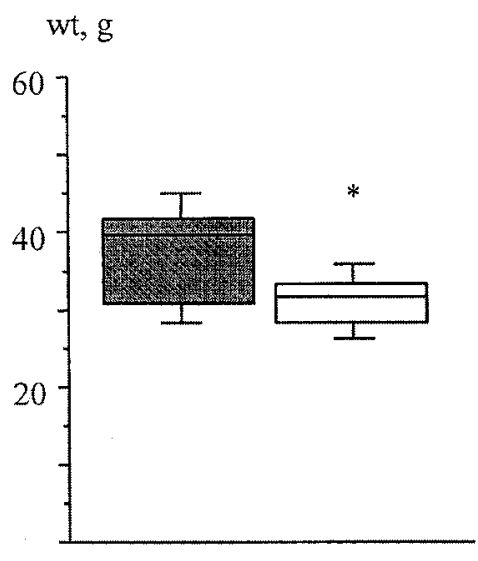

3
B.

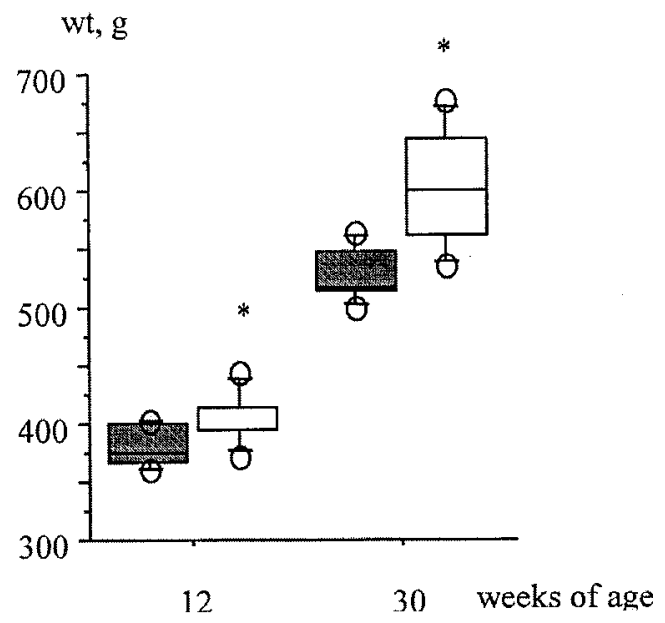

Fig. 1. Effect of the diet deficient in essential fatty acids (EFA) given during the perinatal period on body weight of offspring: (A) 3-week-old rats, and (B) 12- and 30-week-old rats ( $n=7$ rats per group). The results are expressed as box plots, which indicate median, lower and upper quartiles. The whiskers show the 5 th and 95 th percentiles. ${ }^{*}$ Results significantly different from control values $(p<0.05)$. Filled box plots: animals on control diet; open box plots: animals on EFA-deficient diet. (Note the difference in the scale between A and B.)

in rat offspring that persisted into adulthood and would be partly mediated by low neonatal leptin levels.

\section{Changes in leptin levels}

The serum leptin levels during the first week of age were two-fold lower in the offspring of the dams fed the diet enriched in n-3 EFA (n-3 diet) than in those fed the diet containing nine-fold more $n-6$ EFA than n-3 EFA (n-6/n-3 diet). Further increase in the dietary intake of n-6 EFA (n-6 diet) did not elevate the leptin levels in the offspring further (Fig. 2). The lower serum leptin levels in the n-3 group were related to the lower adipose tissue mass and smaller adipocyte size. The data demonstrate that the total intake and the ratio of n-6 to n-3 EFA in the mother's diet modulated neonatal leptin levels. Furthermore, the maternal n-6/n-3 diet promoted increase in body weight, inguinal WAT mass and adipocyte size, and suppressed leptin mRNA levels in WAT in the offspring (3).

\section{Long-term effects of n-3 and n-6 in the mother's diet}

After weaning, all the offspring received the same diet and the serum phospholipid FA composition was similar in adult rats. However, at postnatal week 28 the food intake and the mean body weight were significantly higher in the offspring of the dams fed the n-6/n-3 diet during pregnancy and lactation compared with the other two diet groups (4). The fasting plasma insulin concentration was elevated in both the adult male and female rats of the $n-6 / n-3$ group, but the systolic blood pressure was only increased in the adult male rats. The balance between n-3 and n-6 EFA in the mother's diet seemed to have important long-term effects in the offspring. The long-term effects of changes in neonatal leptin expression could be part of the pathogenesis and should be investigated further.

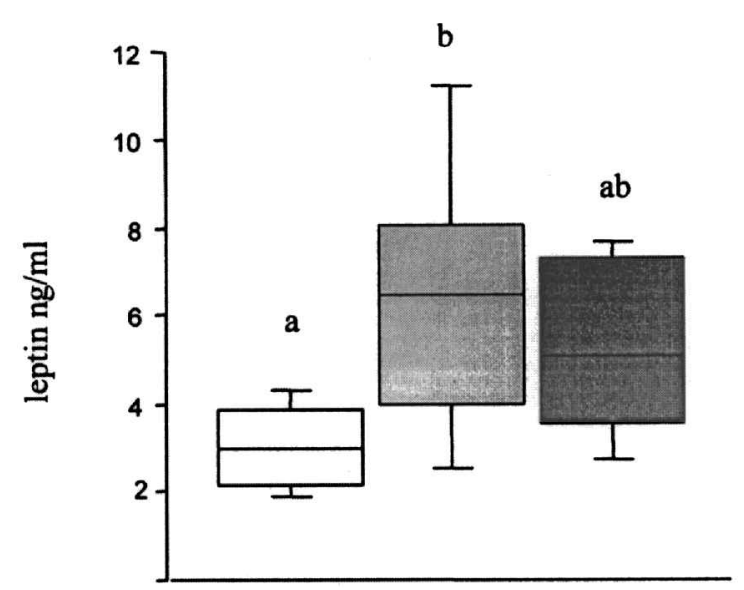

Fig. 2. Effect of different diets on serum leptin levels in rat offspring at 1 week of age ( $n=9$ rats per group). The results are expressed as box plots, which indicate median, lower and upper quartiles. The whiskers show the 5 th and 95 th percentiles. Values with different letters are significantly different $(p<0.05)$. Open box plots: animals on n-3 diet; shaded box plots: animals on $n-6 / n-3$ diet; filled box plots: animals on $n-6$ diet. 
There is also the possibility of direct actions of EFA or its metabolites on gene expression and perinatal metabolism, influencing insulin-glucose homoeostasis, blood pressure and body weight later in life.

This study demonstrated that variations in EFA supplementation during the perinatal period could induce adaptations at the molecular, cellular and biochemical levels, and that these changes may persist into adulthood. These findings may have relevance for the development of diseases in humans.

\section{Articles in Thesis}

1. Korotkova M, Gabrielsson B, Hanson LÅ, Strandvik B: Maternal essential fatty acid deficiency depresses serum leptin levels in suckling rat pups. J Lipid Res 2001;42:35965.

2. Korotkova M, Gabrielsson B, Hanson LÅ, Strandvik B: Maternal dietary intake of essential fatty acids affects adipose tissue growth and leptin mRNA expression in suckling rat pups. Pediatr Res 2002;52:78-84.

3. Korotkova M, Gabrielsson B, Lönn M, et al. Leptin levels in rat offspring are modified by the ratio of linoleic to alpha-linolenic acid in the maternal diet. J Lipid Res 2002; 43: in press.

4. Korotkova M, Holmäng A, Larsson B-M, et al. Perinatal ratio of linoleic to alpha-linolenic acid affects weight, blood pressure and insulin levels in adult rats. Submitted.

The article is based on the author's thesis, which was conducted at the Department of Pediatrics and Department of Clinical Immunology, Göteborg University, Göteborg, Sweden. Defended on June 5, 2002. ISBN: 9I-628-525I-5

Marina Korotkova

Department of Pediatrics, Göteborg University, SE-4I6 85

Göteborg, Sweden

Phone: (46) 3I 34248 33;

FAX: (46) 31 2170 23;

E-mail: Marina.Korotkova@vgregion.se 